\title{
Incremental Case-based Pattern Classifier
}

\author{
Joo-Hwee LIM \\ Institute of Systems Science, National University of Singapore \\ Heng Mui Keng Terrace, Kent Ridge \\ Singapore 0511, Republic of Singapore \\ email: joohwee@iss.nus.sg
}

\begin{abstract}
In this paper, we propose a new Incremental Case-based neural nEtwork (ICE) for pattern classification. We develop the framework upon a case-based learning rule. We show its simplicity, efficiency, and balance between storing specific instances and generalizing exemplars. It also allows nested exemplars to be formed and control of cluster fineness. Finally we compare it with other similar incremental learning algorithms by applying them on off-line handwritten numeral recognition. On the digit database obtained from the Centre for Pattern Recognition and Machine Intelligence, Concordia University, Canada, ICE has attained $87.6 \%$ recognition accuracy on the test set, which is at least as good as other learning algorithms: Instance-Based learning algorithm (85.6\%), Instance-Averaging learning algorithm (87.6\%), Restricted Coulomb Energy network learning algorithm (56.5\%), Back-Propagation learning algorithm (85.8\%). We also observe that the recognition accuracy of ICE improves as more instances are used in the training and even though the number of prototypes (ie. hidden nodes) increases as well, the rate of increment levels off.
\end{abstract}

